\title{
PLANEACIÓN Y RENTABILIDAD FINANCIERA EN EMPRESAS PRESTADORAS DE SERVICIOS DE BOMBEO ELECTROSUMERGIBLE EN PDVSA, VENEZUELA
}

\author{
PLANNING AND FINANCIAL PROFIT ABILITY IN COMP ANIES PROVIDING \\ ELECTROSUMERGIBLE PUMPING SERVICES IN PDVSA, VENEZUELA
}

\author{
Rodrigo Daniel Gámez Pitre ${ }^{1}$ \\ Elizabeth Palma Cardoso ${ }^{2}$ \\ Luis Gerardo Beltrán Villalobos ${ }^{3}$
}

Cómo citar esté artículo: Gámez Pitre, R. D., Palma Cardoso, E. P., \& Beltrán Villalobos, L. G. (2020). Planeación y rentabilidad financiera en empresas prestadoras de servicios de bombeo electrosumergible en PDVSA, Venezuela. Revista Innova ITFIP, 7(1), 78-87

Recibido: Junio de 2020. Aprobado: Diciembre de 2020

\section{Resumen}

El presente artículo tiene por objeto determinar la relación entre la planeación financiera y rentabilidad en empresas prestadoras de servicios de Bombeo Electrosumergible en el Campo Moporo de PDVSA, estado Zulia, Venezuela. Para ello, se llevó a cabo una investigación descriptiva, correlacional con diseño no experimental de campo y transeccional, apoyada en la encuesta como instrumento la cual fue aplicada a diferentes personas en el área financiera y gerencial de las empresas Baker Hughes a GE Company y Schlumberger. La investigación arrojó como resultado que las empresas mencionadas anteriormente consideran el presupuesto de ingresos y egresos en su planificación, sin embargo, tienen problemas en considerar el presupuesto de efectivo al no poder controlar la cantidad que requieren para afrontar las necesidades ordinarias de

\footnotetext{
${ }^{1}$ Profesional en Finanzas y Relaciones Internacionales, Especialista en Gerencia de Mercadeo, Magister en Gerencia empresarial, Doctorante en ciencias gerenciales, Docente ocasional adscrito a la dirección de Investigación y Centro de lenguas de la Universidad de la Guajira, miembro activo del grupo de investigación BIEMARC, rgamez@uniguajira.edu.co, La Guajira, Colombia.

2 Doctorante en Administración Gerencial, Magister en Educación, Especialista en Docencia Universitaria, Líder del grupo de investigación SICOFAS (ITFIP), Coordinadora de investigación de Contaduría Pública, Organizadora de eventos científicos, editora de REVISTA INNOVA ITFIP y libros de investigación., Consejo Arbitral REVISTA ROCA Cuba, epalma@itfip.edu.co. Espinal, Colombia

${ }^{3}$ Lcdo. en Administración de Empresas, MSc en Gerencia Empresarial, Doctorante en Ciencias Gerenciales, profesor ordinario, Universidad Politécnica Territorial de Maracaibo, Investigador adscrito al Programa de Estímulo a la Investigación (PEI). luisbeltran14@gmail.com, Maracaibo, Venezuela.
} 
recursos y no poder incluir las entradas esperadas de efectivo para el periodo financiero especifico, además se concluye que efectivamente las empresas consideran el presupuesto de capital y presupuesto de utilidades para elaborar los planes financieros a largo plazo, del mismo modo se observa que el margen de utilidades y la rotación de activos son calculados por las empresas para el cálculo de la rentabilidad económica, del mismo modo tanto el margen de utilidades como la rotación de activos son estudiados para determinar la rentabilidad financiera. Finalmente se obtuvo que la planeación financiera y la rentabilidad tienen una relación débil en el presente estudio con un valore de correlación de Pearson de 0,36.

Palabras clave: Planeación financiera, rentabilidad, competitivo, presupuesto.

\section{Abstract:}

The purpose of this article is to determine the relationship between financial planning and profitability in companies that provide Electrosubmersible Pumping services in the Moporo Field of PDVSA, Zulia state, Venezuela. For this, a descriptive, correlational research was carried out with a non-experimental field and transectional design, supported by the survey as an instrument which was applied to different people in the financial and managerial areas of the Baker Hughes, GE Company and Schlumberger companies. The investigation showed as a result that the companies mentioned above consider the budget of income and expenses in their planning, however, they have problems in considering the cash budget because they cannot control the amount they require to meet the ordinary needs of resources and cannot include the expected cash inflows for the specific financial period, it is also concluded that the companies actually consider the capital budget and profit budget to prepare long-term financial plans, in the same way it is observed that the profit margin and turnover of assets are calculated by companies to calculate the economic profitability, in the same way both the profit margin and the rotation of assets are studied to determine the financial profitability. Finally, it was obtained that financial planning and profitability have a weak relationship in the present study with a Pearson correlation value of 0.36.

Keywords: Financial planning, profitability, competitive, budget.

\section{Introducción}

En los últimos años el mundo petrolero se ha visto fuertemente afectado debido a la notoria baja en los precios del barril de petróleo, esto ha puesto en una difícil situación a varias economías que dependen en gran medida de la explotación de este recurso natural, es por ello por lo que las empresas de servicios han tenido que revisar sus estrategias con el fin de seguir siendo rentables en tiempos difíciles. En 2014 el barril de petróleo de esta cesta promedió 96,29 dólares, en 2015 49,49 dólares en 2016 40,76 dólares, en 2017 52,37 y en lo que va de 2018 promedia 65.10 dólares por barril (OPEC, 2018). Esto representa una caída importante con respecto al 2014 que sin dudas ha impactado negativamente a aquellas economías con una fuerte dependencia de la importación de petróleo crudo, Latinoamérica es sin duda unas de las regiones más fuertemente afectadas a nivel mundial. 
REVISTA INNOVA ITFIP, 7 (1). 78-87. DIC. 2020

Específicamente en V enezuela la situación no es diferente, al ser un país altamente dependiente de la venta de petróleo crudo ha visto que sus ingresos en moneda extranjera se han disminuido más de la mitad. Según el portal web del Ministerio del Poder Popular de Petróleo (MPPP, 2018), el precio promedio del barril de petróleo crudo venezolano en el año 2014 fue de 88,42 dólares, en 2015 fue de 44,65 dólares, en 2016 fue 35,15 dólares, para el año 2017 el promedio fue de 46,66 y en 2018 promedio 60,55 dólares lo cual igualmente representa una caída importante con respecto al 2014. Esta situación ha repercutido en toda la industria petrolera nacional especialmente en aquellas unidades de explotación operadas 100\% por PDVSA, este es el caso del campo Moporo en la División sur del Lago Trujillo.

El campo Moporo representa uno de los campos Petroleros más importantes del país, este forma parte de la división Sur del Lago Trujillo de PDVSA, su producción diaria se encuentra en el orden de los 70,000 barriles netos. Dado el potencial de producción del campo, en él hacen vida un número considerable de empresas de servicios petroleros, entre ellas las encargadas de los servicios de instalación, puesta en marcha y monitoreo de Bombas Electrosumergibles como Baker Hughes a GE Company y Schlumberger.

Tanto PDVSA como las empresas de servicios antes mencionadas saben que la posibilidad de que entren nuevos competidores está limitada a muy pocas empresas debido a la complejidad tecnológica de este tipo de levantamiento artificial, mientras que la sustitución de la tecnología es poco probable debido a que otros métodos de producción no alcanzarían a cubrir la capacidad productiva del campo. Hoy más que nunca el poder de negociación está en manos de las empresas prestadoras de servicios de Bombeo Electrosumergible y no de PDVSA; sencillamente son muy pocas las empresas que encuentran atractivo hacer negocios bajo las condiciones actuales.

Según Gitman \& Zutter (2012), la rentabilidad es la relación entre los ingresos y los costos generados por el uso de los activos de la compañía (tanto corrientes como fijos) en actividades productivas. Así mismo, "la gestión contable se debe mirar desde la perspectiva de inversión, no de gastos, ya que les permite a las empresas ejercer un control financiero, evitando sanciones y consumos tributarios innecesarios, o en últimas ir al fracaso". (Palma, Alarcón, \& Hernández, 2018)

En general, la rentabilidad no es más que la relación del beneficio o ingreso que produce un activo y el costo de los mismos. Por otro lado, la rentabilidad de una empresa está íntimamente relacionada con los planes establecidos por ella. La planeación financiera es un aspecto importante de las operaciones de la empresa porque brinda rutas que guían, coordinan y controlan las acciones de la empresa para lograr sus objetivos (Gitman \& Zutter, 2012). Aunque otros autores, plantean que la planeación financiera consiste en minimizar el riesgo aprovechando las oportunidades y los recursos financieros, decidir anticipadamente las necesidades de dinero y su correcta aplicación buscando su mejor rendimiento y máxima seguridad, siendo el sistema presupuestario es su herramienta más importante (Galeano \& Tinjaca, 2012). 
Por lo anterior, se destaca la planeación financiera como proceso fundamental para cualquier empresa, dado que persigue el logro de los objetivos empresariales a través de proyecciones de ventas, ingresos y activos con base en las estrategias de producción y mercadotecnia. Basados en su planeación financiera y rentabilidad, las empresas de servicios antes mencionadas, se han visto obligadas a reducir considerablemente sus operaciones en V enezuela en los últimos años y por primera vez en muchos años, viéndose obligadas a reevaluar sus planes financieros, en particular, la evaluación del efectivo disponible al corto o mediano plazo.

Si a las caídas en los precios del petróleo iniciada a finales del 2014, se le suma que desde hace unos años incluso cuando el barril de petróleo rondaba los 100 dólares, PDVSA no liquidaba sus cuentas por pagar a tiempo, se encuentra un escenario sumamente peligroso para la estabilidad económica de las empresas que prestan servicios petroleros a la industria petrolera venezolana, y esta inestabilidad puede ser transmitida a PDVSA ya que al no tener empresas dispuestas a hacer los servicios de bombeo electrosumergible, su producción podría verse muy comprometida.

Por lo anterior, de mantenerse o de agravarse la situación de pagos de PDVSA las empresas de servicios bajo los planes financieros actuales tendrían una rentabilidad tan comprometida que pudiera significar el cierre de sus puertas en el país dando como resultado una caída en la producción de petróleo del campo Moporo que sin dudas impactaría la producción nacional y por tanto el acceso a divisas del país. Ante las razones expuestas, se planteó como objetivo determinar la relación entre la planeación financiera y rentabilidad de las empresas prestadoras de servicios de Bombeo Electrosumergible en el Campo Moporo de la División Sur del Lago Trujillo de PDVSA, estado Zulia, Venezuela.

\section{Metodología}

Se empleó un diseño de campo, descriptivo y correlacional, para el análisis de la variables, Planeación Financiera por medio de las dimensiones Planeación a corto plazo (Presupuesto de egresos, Presupuesto de ingresos, Presupuesto de efectivo) y Planeación a largo plazo (Presupuesto de capital, Presupuesto de utilidades); y la variable Rentabilidad, fue abordada por las dimensiones Rentabilidad económica (Margen de utilidades, Rotación de activos), Rentabilidad financiera (Rentabilidad de las ventas, Apalancamiento financiero), y Factores competitivos (Grado de rivalidad entre competidores, Amenaza de nuevos competidores, Presión de productos sustitutos, Poder de negociación de consumidores, Poder de negociación de proveedores). Por medio de un censo poblacional, se seleccionaron como unidades de información a 12 sujetos responsables del área de finanzas (Gerentes, lideres, contralor, administradores y analistas) de las dos únicas empresas que prestan el servicio de bombeo electrosumergible en el campo Moporo de PDVSA, Baker Huges a GE company ( $\mathrm{n}=$ 5), y Schlumberger $(n=7)$. 
REVISTA INNOVA ITFIP, 7 (1). 78-87. DIC. 2020

Durante el periodo marzo 2016 - enero 2018, se realizaron encuestas por medio de un cuestionario de 42 interrogantes, con cinco (5) alternativas de respuestas, que fueron Siempre (S), Casi Siempre (CS), Algunas V eces (A V), Casi Nunca (CN), y Nunca (N), a las cuales se les aplicó una puntuación ponderada de 5, 4, 3, 2 y 1 respectivamente, para establecer así una escala tipo Lickert. El cuestionario fue validado por cinco (5) expertos en las áreas objeto de estudio, su confiabilidad fue determinada por una prueba piloto aplicada a sujetos de empresas con similares características a las del presente estudio, a estos resultados preliminares se aplicó el coeficiente Alpha-Cronbach, que presentó una alta confiabilidad $(\alpha=0,70)$ (Hernández, Fernández, \& Baptista, 2010).

Por medio de la valoración de las opciones se cuantificó la opinión general de los encuestados, para finalmente categorizar la presencia de las características estudiadas por medio de un rango de valores establecido, siendo estos específicamente Muy bueno (4,5 - 5), Bueno $(4-4,4)$, Regular (3 - 3,9), Malo (2-2,9) y Muy malo (1 - 1,9) (Hernández et al., 2010).

Se aplicó estadística descriptiva como frecuencias relativas y la media aritmética de los valores ponderados, para analizar los indicadores y las dimensiones estudiadas en cada variable. Adicionalmente se realizó un análisis de correlación de Pearson, para observar si existe una relación entre la Planificación financiera y la Rentabilidad en las empresas estudiadas. Todos los análisis fueron realizados por medio del programa SPSS Statistics 24.0.

\section{Resultados y Discusión}

La planeación financiera mostró según su dimensión Planeación a corto plazo que el indicador Presupuesto de egresos obtuvo un 58,3\% de los encuestados que respondió siempre y un 30,6\% respondió casi siempre con una media ponderada de 4,5 que lo ubica en la categoría de muy buena (Tabla 1). Efectivamente el presupuesto de egresos es tomado en cuenta para elaborar la planeación financiera a corto plazo lo que está en línea con lo expuesto por Ortega (2008), quien afirma que el presupuesto de egresos es una herramienta fundamental para la planeación a corto plazo y el mismo debe considerar el presupuesto de inventario, producción, costos de producción y compras, así como el presupuesto de costos de distribución y administración, el presupuesto de impuesto sobre la renta, el de aplicación de las utilidades y el presupuesto de otros egresos.

El indicador presupuesto de ingresos, mostró un $61,1 \%$ de los encuestados en la opción siempre, y un $25 \%$ respondió casi siempre, para una media ponderada de 4,4 de categoría buena (Tabla 1). Esto significa que efectivamente el presupuesto de ingresos es tomado en cuenta para elaborar la planeación financiera a corto plazo, cumpliendo así el computo anticipado de los ingresos, como primer paso en la implantación de todo programa presupuestal (Ortega, 2008).

El presupuesto de efectivo obtuvo un 33,3\% de los encuestados que respondió siempre, y el 36,1\% respondió casi siempre, para una media de 3,9 y una categoría de regular (Tabla 1), lo cual cumple mediamente lo expuesto por Ortega (2008), quien afirma que el presupuesto de 
efectivo controla la cantidad de efectivo que la empresa debe tener siempre disponible para afrontar las necesidades ordinarias de recursos para efectuar las erogaciones correspondientes; por lo anterior las empresas encuestadas frecuentemente no elaboran el presupuesto de efectivo.

En general la dimensión de Planeación corto plazo mostró en promedio 4,3 lo cual muestra una categoría buena, la cual señala que los planes financieros a corto plazo reflejan los resultados que se esperan a partir de acciones programadas a una fecha próxima, generalmente de uno a dos años (Ortega, 2008).

Tabla 1. Frecuencias relativas de las respuestas de los encuestados y valor de la media ponderada para los indicadores y dimensión Planeación a corto plazo.

\begin{tabular}{cccccccc}
\hline Indicadores & Siempre & $\begin{array}{c}\text { Casi } \\
\text { Siempre }\end{array}$ & $\begin{array}{c}\text { Algunas } \\
\text { Veces }\end{array}$ & $\begin{array}{c}\text { Casi } \\
\text { Nunca }\end{array}$ & Nunca & Categoría \\
\hline $\begin{array}{c}\text { Presupuesto de } \\
\text { egresos } \\
\begin{array}{c}\text { Presupuesto de } \\
\text { ingresos }\end{array}\end{array}$ & 58,3 & 30,6 & 11,1 & 0 & 0 & 4,5 & Muy Buena \\
$\begin{array}{c}\text { Presupuesto de } \\
\text { efectivo }\end{array}$ & 33,3 & 36,1 & 22,2 & 2,8 & 5,6 & 3,9 & Regular \\
\hline $\begin{array}{c}\text { Planeación a } \\
\text { corto plazo }\end{array}$ & $\mathbf{5 0 , 9}$ & $\mathbf{3 0 , 6}$ & $\mathbf{1 4 , 8}$ & $\mathbf{1 , 9}$ & $\mathbf{1 , 9}$ & $\mathbf{4 , 3}$ & Buena \\
\hline
\end{tabular}

Fuente: Gámez, Palma \& Beltrán (2020)

La planeación financiera a largo plazo mostró en su indicador Presupuesto de capital y Presupuesto de utilidades, que los mayores porcentajes de respuesta de los encuestados estuvieron entre las opciones de respuesta Siempre y casi siempre con más de un $70 \%$ entre las dos opciones de respuesta, para obtener así medias de 4,2 y 4,4 respectivamente, con una categoría de bueno, que se refleja en igual categoría para toda la dimensión (Tabla 2). Efectivamente el presupuesto de capital y de utilidades se cumple, para elaborar la planeación financiera a largo plazo; el presupuesto de capital pronostica las inversiones en propiedades, edificios y equipo principal que realizará la empresa en los próximos años, mientras que el presupuesto de utilidades se utiliza para determinar la contribución tomando en cuenta los ingresos y gastos. El plan financiero a largo plazo refleja las estrategias en términos de operaciones y acciones en que la empresa deberá elaborar a fin de lograr sus objetivos planteados en un periodo de uno a cinco años (Ortega, 2008; Calderón, 2018). 
REVISTA INNOVA ITFIP, 7 (1). 78-87. DIC. 2020

Tabla 2. Frecuencias relativas de las respuestas de los encuestados y valor de la media ponderada para los indicadores y dimensión Planeación a largo plazo.

\begin{tabular}{cccccccc}
\hline Indicadores & Siempre & $\begin{array}{c}\text { Casi } \\
\text { Siempre }\end{array}$ & $\begin{array}{c}\text { Algunas } \\
\text { Veces }\end{array}$ & $\begin{array}{c}\text { Casi } \\
\text { Nunca }\end{array}$ & Nunca & Categoría \\
\hline $\begin{array}{c}\text { Presupuesto de } \\
\text { capital }\end{array}$ & 44,4 & 33,3 & 19,4 & 2,8 & 0 & 4,2 & Buena \\
$\begin{array}{c}\text { Presupuesto de } \\
\text { utilidades }\end{array}$ & 50 & 44,4 & 5,6 & 0 & 0 & 4,4 & Buena \\
\hline $\begin{array}{c}\text { Planeación a } \\
\text { largo plazo }\end{array}$ & $\mathbf{4 7 , 2}$ & $\mathbf{3 8 , 9}$ & $\mathbf{1 2 , 5}$ & $\mathbf{1 , 4}$ & $\mathbf{0 , 0}$ & $\mathbf{4 , 3}$ & Buena \\
\hline
\end{tabular}

Fuente: Gámez, Palma \& Beltrán (2020)

La variable Rentabilidad, a nivel de su dimensión Rentabilidad económica, mostró en sus indicadores Margen de utilidades y Rotación de activos, que los mayores porcentajes de respuestas estuvieron en las opciones siempre y casi siempre, que les categoriza en un nivel bueno (Tabla 3). El margen de utilidades mide la unidad monetaria de beneficio obtenido por unidad monetaria vendida, esta razón expresa un beneficio a alcanzar a través de una serie de acciones empresariales que ayuden a su incremento (Ortega, 2008); por su parte la rotación de activos es una de las razones que genera la rentabilidad económica, e identifica la efectividad con la que se utilizan los activos de la empresa, potenciando así la tasa con que la empresa remunera a la totalidad de los recursos (inversiones o activos) utilizados en su explotación, midiendo así la capacidad del activo de la empresa para generar beneficios (Eslava, 2010).

Tabla 3. Frecuencias relativas de las respuestas de los encuestados y valor de la media ponderada para los indicadores y dimensión Rentabilidad económica.

\begin{tabular}{cccccccc}
\hline Indicadores & Siempre & $\begin{array}{c}\text { Casi } \\
\text { Siempre }\end{array}$ & $\begin{array}{c}\text { Algunas } \\
\text { Veces }\end{array}$ & $\begin{array}{c}\text { Casi } \\
\text { Nunca }\end{array}$ & Nunca & Categoría \\
\hline Margen de utilidades & 33,3 & 47,2 & 19,4 & 0 & 0 & 4,1 & Buena \\
Rotación de activos & 36,1 & 36,1 & 27,8 & 0 & 0 & 4,1 & Buena \\
\hline $\begin{array}{c}\text { Rentabilidad } \\
\text { económica }\end{array}$ & $\mathbf{3 4 , 7}$ & $\mathbf{4 1 , 7}$ & $\mathbf{2 3 , 6}$ & $\mathbf{0}$ & $\mathbf{0}$ & $\mathbf{4 , 1}$ & Buena \\
\hline
\end{tabular}

Fuente: Gámez, Palma \& Beltrán (2020)

La dimensión de Rentabilidad financiera dentro de la variable de Rentabilidad, igualmente mostró en sus indicadores Rentabilidad de las ventas y apalancamiento financiero que los mayores porcentajes de los encuestados respondieron siempre y casi siempre, lo que 
resultó en medias ponderadas de 4,7 y 4,4 para cada indicador respectivamente (Tabla 4). La Rentabilidad de ventas obtuvo un muy buen nivel, que significa un cumplimiento efectivo de esta para la planeación financiera lo que está en línea con lo expuesto por Eslava (2010), quien afirma que esta rentabilidad representa la ganancia obtenida por la empresa después de realizar sus ventas e implica la utilidad neta derivada de las actividades de venta en el mercado. El apalancamiento financiero al obtener una categoría buena, se muestra como un factor tomado en cuenta para elaborar la planeación financiera, haciendo uso de los recursos ajenos para elevar el rendimiento financiero de los accionistas (Eslava, 2010).

La Rentabilidad financiera es una razón que mide la capacidad de la empresa para remunerar a sus propietarios, representados para ellos en última instancia el coste de oportunidad de los fondos que mantienen invertidos en la empresa y posibilita la comparación, al menos en principio con los rendimientos de otras inversiones alternativas (Eslava, 2010).

Tabla 4. Frecuencias relativas de las respuestas de los encuestados y valor de la media ponderada para los indicadores y dimensión Rentabilidad financiera.

\begin{tabular}{cccccccc}
\hline Indicadores & Siempre & $\begin{array}{c}\text { Casi } \\
\text { Siempre }\end{array}$ & $\begin{array}{c}\text { Algunas } \\
\text { Veces }\end{array}$ & $\begin{array}{c}\text { Casi } \\
\text { Nunca }\end{array}$ & Nunca & Categoría \\
\hline $\begin{array}{c}\text { Rentabilidad de } \\
\text { ventas }\end{array}$ & 72,2 & 22,2 & 5,6 & 0 & 0 & 4,7 & $\begin{array}{c}\text { Muy } \\
\text { Buena }\end{array}$ \\
$\begin{array}{c}\text { Apalancamiento } \\
\text { financiero }\end{array}$ & 58,3 & 25 & 13,9 & 2,8 & 0 & 4,4 & Buena \\
\hline $\begin{array}{c}\text { Rentabilidad } \\
\text { financiera }\end{array}$ & $\mathbf{6 5 , 3}$ & $\mathbf{2 3 , 6}$ & $\mathbf{9 , 8}$ & $\mathbf{1 , 4}$ & $\mathbf{0}$ & $\mathbf{4 , 6}$ & $\begin{array}{c}\text { Muy } \\
\text { Buena }\end{array}$ \\
\hline
\end{tabular}

Fuente: Gámez, Palma \& Beltrán (2020)

La dimensión Factores competitivos (variable Rentabilidad), mostraron una tendencia similar a las dimensiones o características anteriores, los encuestados se inclinaron en su mayoría a las opciones de respuesta siempre y casi siempre, sin embargo, se obtuvieron distintos niveles entre los indicadores, como el grado de rivalidad entre competidores, Amenaza de nuevos competidores, y el poder de negociación de proveedores obtuvieron una categoría buena, mientras que la Presión de productos sustitutos y el Poder de negociación de consumidores obtuvieron una categoría regular (Tabla 5).

Lo anterior indica que los factores en una categoría buena, son tomados en cuenta para identificar condiciones competitivas, lo que está en línea con lo expuesto por Porter (2015), quien afirma que las empresas mantienen relaciones y competencia dentro de un mismo sector industrial, dado que ofrecen el mismo tipo de producto; mientras que aquellos en categoría regular muestran deficiencias en su implementación como factores de competencia considerados, destacando la poca atención que le es prestada a productos sustitutos que podrían representar un desplazamiento del negocio propio de las empresas estudiadas. 
En general la dimensión Factores competitivos dentro de la variable Rentabilidad, mostró una tendencia favorable con una media de 4 con una categoría buena, lo cual se encuentra en línea con lo expuesto por Porter (2015), quien señala que la competitividad puede afectarse por factores o elementos externos, propios de las capacidades de los competidores, los cuales constituyen fuerzas competitivas, que pueden favorecer o afectar la posición de la empresa frente a sus competidores y en función de ello representar ventajas competitivas.

Tabla 5. Frecuencias relativas de las respuestas de los encuestados y valor de la media ponderada para los indicadores y dimensión Factores competitivos.

\begin{tabular}{|c|c|c|c|c|c|c|c|}
\hline Indicadores & Siempre & $\begin{array}{c}\text { Casi } \\
\text { Siempre }\end{array}$ & $\begin{array}{l}\text { Algunas } \\
\text { Veces }\end{array}$ & $\begin{array}{c}\text { Casi } \\
\text { Nunca }\end{array}$ & Nunca & & Categoría \\
\hline $\begin{array}{l}\text { Grado de rivalidad entre } \\
\text { los competidores }\end{array}$ & 50 & 38,9 & 11,1 & 0 & 0 & 4,4 & Buena \\
\hline $\begin{array}{l}\text { Amenaza de nuevos } \\
\text { competidores }\end{array}$ & 41,7 & 30,5 & 16,7 & 8,3 & 2,8 & 4 & Buena \\
\hline $\begin{array}{l}\text { Presión de productos } \\
\text { sustitutos }\end{array}$ & 38,9 & 27,8 & 25 & 5,6 & 2,8 & 3,9 & Regular \\
\hline $\begin{array}{l}\text { Poder de negociación de } \\
\text { consumidores }\end{array}$ & 33,3 & 30,5 & 22,2 & 13,9 & 0 & 3,9 & Regular \\
\hline $\begin{array}{l}\text { Poder de negociación de } \\
\text { proveedores }\end{array}$ & 33,3 & 41,7 & 22,2 & 0 & 2,8 & 4 & Buena \\
\hline Factores competitivos & 33,3 & 36,1 & 22,2 & 7,0 & 1,4 & 4 & Buena \\
\hline
\end{tabular}

Fuente: Gámez, Palma \& Beltrán (2020)

El análisis de correlación en función de todos los indicadores y dimensiones estudiadas, arrojo un coeficiente de 0,36 que muestra una correlación positiva débil entre la variable Planificación financiera y Rentabilidad, por lo que no se evidencia relación entre estas variables en las empresas de prestadoras de servicios de bombeo electrosumergible del campo Moporo de la división sur del lago Trujillo de PDVSA.

\section{Conclusiones}

La planificación financiera y la rentabilidad muestran elementos que se encuentran presentes, tomados en cuenta dentro de las empresas prestadoras de servicios de Bombeo Electrosumergible en el Campo Moporo de la División Sur del Lago Trujillo de PDVSA, así 
como existe una débil relación entre estas variables, de modo que una buena planeación financiera no implica una buena rentabilidad ni viceversa en las empresas estudiadas.

Se recomienda que las empresas prestadoras de servicios de Bombeo Electrosumergible diseñen y pongan en práctica estrategias destinadas a impedir la penetración de otras empresas que vendan productos alterativos, ya que el mercado de este mecanismo es altamente susceptible a ser ocupado por otro tipo de componente como el bombeo mecánico o el bombeo de cavidad progresiva, siendo un factor potencialmente presente y al cual se le presta poca atención por parte de estas empresas.

\section{Referencias}

Calderón Saque, E. A. (2018). Educación económica y financiera, una solución a la exclusión. Revista Innova ITFIP, 3(1), 44-51. Recuperado a partir de http://revistainnovaitfip.com/index.php/innovajournal/article/view/38

Eslava, J. (2010). Las claves del análisis económico-financiero de la empresa. España: ESIC Editorial.

Galeano, P., \& Tinjacá, J. (2012). Planeación estratégica financiera, el camino al éxito empresarial. Colombia: Fundación Universitaria San Martin.

Gitman, L., \& Zutter, C. (2012). Principios de Administración Financiera (Vol. Décimo Segundo).

Hernández, R., Fernández, C., \& Baptista, P. (2010). Metodología de la. Ciudad de México: Mc Graw Hill.

MPPP. (2018). Boletín estadístico de precios del petróleo [Gubernamental]. Recuperado de http://www.minpet.gob.ve/index.php/es-es/

OPEC. (2018). OPEC Basket price [Official]. Recuperado de https://www.opec.org/opec_web/en/data_graphs/40.htm

Ortega, A. (2008). Planeación financiera estratégica (segunda edición,). México-México: Editorial McGraw-Hill Interamericana Editores.

Palma Cardoso, E., Alarcón Linares, A. F., \& Hernández Pava, E. A. (2018). Diseño de un sistema informático (software) para automatizar los procesos contables en el sector mecánico automotriz del régimen simplificado. Revista Innova ITFIP, 2(1), 62-70. Recuperado a partir de http://revistainnovaitfip.com/index.php/innovajournal/article/view/29

Porter, M. E. (2015). Ventaja competitiva: creación y sostenimiento de un desempeño superior (segunda edición). México: Grupo Editorial Patria 